\title{
EL NUEVO PARADIGMA UNIVERSITARIO DE ACCESO UNIVERSAL: SU ORIGEN, CARACTERÍSTICAS Y ALCANCES EN RELACIÓN A LA ENSENAANZA DEL DERECHO
}

\author{
NEW PARADIGM OF UNIVERSAL ACCESS TO UNIVERSITY: \\ ORIGIN, CHARACTERISTICS AND SCOPE IN THE FIELD OF LEGAL \\ EDUCATION
}

\section{JOHANN BENFELD E.*}

\begin{abstract}
RESUMEN: En este ensayo, desde una perspectiva teórica, se afirma que el nuevo modelo universitario de acceso universal tiende a ejercer una influencia en el modo en que se enseña y aprende el derecho. Dicha influencia ha sido bien percibida por los teóricos del mundo anglosajón, quienes han mostrado, desde la década de los 70 en adelante, cómo las cuestiones referidas a la diversidad; las tasas de retención y egreso, y la vinculación entre teoría y práctica tensionan el sistema tradicional de enseñanza del derecho, debido, por una parte, a la necesidad de cambios y, por otra, a los riesgos que ellos suponen. Esta tensión dentro del sistema universitario norteamericano, progresivamente, ha comenzado a expandirse por todo occidente. Por lo mismo, la descripción y análisis que se ofrece en este trabajo sobre el estado actual de la cuestión también puede ayudar a entender las oportunidades, desafíos y riesgos para la enseñanza del derecho en Chile dentro del nuevo paradigma universitario.
\end{abstract}

Palabras clave: Educación superior, enseñanza del derecho, diversidad, tasas de retención, teoría y práctica.

ABSTRACT: This paper claims that from a theoretical perspective the new university model of universal access influences the way in which law is taught and learned. This influence has been well perceived by theorists of the Anglo-American sphere within their university systems. From the 70's onwards they have shown that the need for change and the risks it poses is a consequence of the pressure placed on the legal education system by issues related to diversity, retention rates, graduation and the link between theory and practice. This tension has gradually expanded through the West, including Chile. Therefore, the description and analysis of the current state of the subject presented in this paper can help us to understand the opportunities, challenges and risks of legal education within the new university paradigm.

Key words: Higher education, legal education, diversity, retention rates, theory and practice.

\footnotetext{
* Profesor de Teoría y Filosofía del Derecho de la Pontificia Universidad Católica de Valparaíso. Abogado. Licenciado en Ciencias Jurídicas y Filosofía por la Pontificia Universidad Católica de Valparaíso. Doctor en Derecho por la Universidad de Salamanca, España. Dirección postal: Facultad de Derecho, Pontificia Universidad Católica de Valparaíso, Avda. Brasil 2950, Valparaíso, Chile. Correo electrónico: johann.benfeld@pucv.cl
} 


\section{CONSIDERACIONES PRELIMINARES}

En un erudito artículo de 2006 Alejandro Guzmán llamaba la atención sobre el carácter altamente conservador de la enseñanza del derecho en Chile. En casi 200 años, tanto los curricula de las escuelas de derecho como la metodología de la enseñanza de los programas académicos apenas habían experimentado cambios significativos ${ }^{1}$. La situación descrita por Guzmán, según refiere el propio autor, cambió radicalmente desde 1981. A partir de ese año, no solo hubo un aumento progresivo y significativo de las escuelas de derecho nacionales, sino también un proceso de reforma estructural y curricular de las mismas que se mantiene hasta nuestros días.

El proceso de reforma, por cierto, no obedeció a un movimiento interno de las propias facultades o escuelas de derecho nacionales, sino a una exigencia de las circunstancias políticas, históricas y sociales. En efecto, en 1981 se dictó en nuestro país el decreto con fuerza de ley n ${ }^{\circ} 1$ (del Ministerio de Educación), que no solo modificó el escenario de la enseñanza del derecho en términos cuantitativos, sino también cualitativos al introducir, tal vez incluso sin intención, un cambio de paradigma en el sistema de la educación terciaria: las universidades chilenas, vía decreto, abandonaban el clásico modelo de educación superior de élite para abrazar el nuevo paradigma de acceso universal.

El cambio de paradigma, a su vez, no fue una creación nacional. Desde la década de los años 70 las universidades de los Estados Unidos de América y Europa Occidental habían comenzado a transitar dicho camino como una deriva natural de las exigencias propias de las sociedades industrializadas de carácter democrático. En dicho contexto, las reformas fueron lentas y graduales. Primero se masificó el acceso a las universidades, cuestión que significó que grupos históricamente excluidos de la educación superior pudiesen por primera vez acceder a este tipo de formación. Luego, la diversidad de los estudiantes hizo necesario reformular los métodos de enseñanza y aprendizaje a fin de lograr que aquellos no solo tuviesen la oportunidad real de acceder a la universidad sino también de permanecer y egresar de la misma. Finalmente, el cada vez mayor número de graduados disponibles en los mercados económicos generó una relación más estrecha entre las universidades y el mercado laboral, cuestión que derivó en un cualitativo cambio de enfoque respecto a los logros de aprendizaje esperados por los nuevos alumnos, pasando de un modelo basado en objetivos a uno sustentado en competencias.

Especialmente en los países dentro de los cuales primeramente obró el cambio de paradigma, las cuestiones sobre su origen, ideas rectoras y alcances han sido objeto de una copiosa literatura científica. Y, en la medida en que el fenómeno se expandió y sumó nuevos actores allende las fronteras de los Estados Unidos de América y Europa Occidental, los estudios y la literatura a ellos asociada se ha tornado prácticamente inabarcable. En nuestro país las investigaciones referidas a este tema se han concentrado en las áreas de la pedagogía, psicología, sociología e incluso en los dominios de la politología y, tomando en consi-

\footnotetext{
1 A propósito del "canon" de los estudios jurídicos en Chile, Guzmán escribe: "Como puede apreciarse, el canon de las asignaturas quedó sustancialmente constituido hacen ya cien años, en 1902; pero fue en la década de los años veinte que empezó a expandirse en intensidad, cuando en 1924 Derecho comercial pasó de uno a dos años; en 1928 Derecho civil pasó de tres a cuatro años; y en 1929 Derecho procesal pasó de dos a tres años. Terminó de hacerlo en 1946, al pasar Derecho Penal de uno a dos años; y Derecho Procesal, de tres a cuatro años". GUZMÁn (2006) p. 338.
} 
deración las particularidades de nuestra realidad, han intentado comprender el fenómeno en toda su complejidad ${ }^{2}$.

Desgraciadamente, a la reflexión general sobre el cambio de paradigma universitario no siempre le ha sucedido una particular sobre el impacto que dicho cambio ha comenzado a ejercer en las diferentes carreras universitarias. Esto, pensamos, ha ocurrido con la enseñanza del derecho en nuestro país. Por cierto, no afirmamos que los temas de enseñanza, didáctica, metodología de la enseñanza y la investigación jurídica, etc., no hayan sido abordados. De hecho hay una abundante bibliografía dedicada al respecto. Sin embargo, lo que no existe, hasta donde llega nuestro conocimiento, es una reflexión en torno a la manera en que el cambio de paradigma universitario (el modelo de acceso universal) ha comenzado a impactar la estructura, organización, didáctica y diseño curricular ${ }^{3}$. En otras palabras, lo que no se ha tratado adecuadamente es la forma en que la diversidad de los nuevos alumnos; las exigencias de las tasas de retención y egreso y las exigencias competenciales que el mundo laboral reclama de las escuelas de derecho ha impulsado un conjunto de reformas estructurales y curriculares de las cuales las propias facultades y escuelas de derecho se han hecho cargo pero de una manera, de momento, bastante acrítica.

2 En tal sentido, los trabajo de José Joaquín Brunner referidos a las transformaciones de la universidad en la era global, los cambios de paradigma universitario, los riesgos y desafíos de la educación superior en contextos de mercados económicamente libres, junto a los análisis comparados del modelo universitario chileno frente a otros paradigmas de educación superior, constituyen, por cierto, verdaderos aportes a la discusión y una buena guía para las futuras políticas públicas sobre el particular (una completa bibliografía de los trabajos de Brunner está disponible en su sitio web: http://www.brunner.cl). Otro tanto se puede decir de los esfuerzos desplegados el último tiempo por el Centro de Estudios de Políticas y Prácticas en Educación y, especialmente de las contribuciones de Andrés Bernasconi sobre los nuevos paradigmas universitarios y las transformaciones estructurales y funcionales que los componentes de las universidades (profesores y estudiantes) han experimentado en el último tiempo (para revisar las aportaciones del Centro de Estudios de Políticas y Prácticas en educación como asimismo la bibliografía particular de Bernasconi- véase: http://www.ceppe.cl/home). Sobre el trasfondo político-económico de las reformas del sistema de educación superior chileno y sus proyecciones también se ha escrito de forma prolija. Espinoza (2005). Estas contribuciones, como las de otros muchos profesionales del mundo de la educación, muestran que nuestro país no ha permanecido inmune a los cambios de paradigma que ha experimentado la educación superior en el mundo occidental.

3 En la literatura jurídica nacional de los últimos años es posible encontrar una constelación de textos que abordan los temas generales de la enseñanza del derecho y la didáctica jurídica. Algunos tratan sobre la enseñanza del derecho circunscrita a los cursos de dogmática jurídica especializada [AgUdelo (2000); CAMPUSANO (2002); Cattán (2002); Alvear (2002); Escandón (2006); Amunátegui (2007); Calvinho (2009)]; otros, en cambio, han ampliado el horizonte de la discusión reflexionando sobre la importancia del estudio de las humanidades en la formación de los abogados [Cárcamo (2000, 2002); Valenzuela (2002); Peseta (2008) (Solari (2015)]. También se ha reparado en la profesión de abogado como criterio modelador de la enseñanza del derecho [Manning, (1972); Abramovich, (1999), Shakhtur (2007/2008); Aróstica, (2009)]. El profesor de derecho ha sido, en algún trabajo, objeto de estudio por parte de la doctrina ya en su dimensión personal ya en su responsabilidad en la elección de los métodos adecuados para la enseñanza del derecho [SQUELLA (1995); Jiménez (2011); Devoto (2014)]. Finalmente, también es posible encontrar algunos trabajos que tratan el tema de la enseñanza y el aprendizaje del derecho en términos generales [CAPrile (2000); Devoto (2012); TAeli et al. (2014)]. En todos estos trabajo, sin embargo, el tema del cambio de paradigma universitario es simplemente supuesto, pero jamás abordado como criterio interpretativo de las reformas sobre la enseñanza del derecho. $\mathrm{Y}$, aun cuando algunos textos tienen como trasfondo el cambio de paradigma universitario [Coloma (2005, 2006); Coloma y Agüero (2012); Guzmán (2006); Lazo (2011); Solari (2012)]; en otro lugar, hemos mostrado mediante el estudio y análisis de la bibliografía científica sobre el particular (desde el año 1968 hasta el presente) que, precisamente, lo que se echa en falta en la discusión nacional es una reflexión sobre el contexto en el que las reformas curriculares y metodológicas cobran sentido BENFELD (2016). 
El presente ensayo no pretende llenar el vacío que denuncia, pero sí esclarecer los elementos teóricos involucrados en el trasfondo de las reformas a fin de suscitar una discusión dentro de la comunidad jurídica sobre el sentido y alcance del nuevo contexto de la educación superior en relación a la enseñanza del derecho. Para tal efecto, a partir de una selección de textos, tomados en su gran mayoría del contexto anglosajón, intentaremos esclarecer de qué manera las ideas componentes o estructurales del nuevo sistema de educación terciaria de acceso universal influyen en la formación de los futuros abogados. En primer lugar, abordamos de manera breve el tránsito desde el modelo de las universidades de élite a las universidades de acceso universal. Entendido como un movimiento propio de los regímenes democráticos mostraremos cómo estas ideas no solo afectan a la composición de los alumnos sino también a la de los claustros académicos. Luego, precisaremos algunas de las ideas estructurales del nuevo modelo: diversidad; retención y egreso y las relaciones entre el mercado laboral y el quehacer universitario. Todas estas ideas las expondremos en relación a la enseñanza del derecho a fin de determinar en qué medida el cambio de modelo cumple las expectativas de los futuros profesionales. Finalmente, a modo de consideraciones de término, mostraremos algunas de las oportunidades, desafíos y riesgos que el nuevo paradigma universitario comporta para la enseñanza del derecho.

\section{DE LAS UNIVERSIDADES DE ÉLITE A LAS UNIVERSIDADES DE ACCESO MASIVO Y DE LAS UNIVERSIDADES DE ACCESO MASIVO A LAS DE ACCESO UNIVERSAL}

Bill Johnston, investigador del Centro para la Práctica Académica de la Universidad de Strathclyde, Glasgow, publicó el año 2010 un pequeño libro titulado The First Year at University. Teaching Students in Transition. El libro, como su título indica, es una investigación sobre el proceso que viven los jóvenes estudiantes en su primer año de universidad. Y, aunque la obra está preferentemente enfocada en los alumnos universitarios de primer año en el contexto del mundo anglosajón, hace suya una clave interpretativa del fenómeno que es exorbitante al horizonte en el cual se presenta: el cambio de modelo universitario.

En el nuevo modelo, la universidad no es más un centro de élite reservado para un fragmento mínimo de la población sino un espacio de aprendizaje masivo y continuo. Este cambio, cada vez más perceptible y generalizado en todo Occidente, plantea un desafío para la sociedad y el sistema universitario en su conjunto. Johnston advierte que se trata de un proceso irreversible y que por lo mismo, "la sociedad del futuro inmediato estará caracterizada por una experiencia universitaria que va más allá de los viejos modelos de 'élite' e incluso de su más reciente representación del 'acceso masivo'. No está claro qué formas pedagógicas asumirá, pero debemos actuar para anticipar los cambios y crear nuevas formas congeniales con lo mejor de los valores tradicionales y las ideas contemporáneas”“.

El cambio de paradigma del que habla Johnston comenzó, en los Estados Unidos de Norteamérica y en Europa Occidental, hace más de cuatro décadas. Uno de los primeros teóricos en advertir el fenómeno y su posible evolución fue Martin Trow en su Problem in the Transition from Elite to Mass Higher Education de 1973. En este texto clásico Trow

\footnotetext{
4 JohNSON (2010) p. 112.
} 
distingue tres fases ideales en el desarrollo de los sistemas universitarios occidentales: un sistema de élite, uno de acceso masivo y otro de acceso universal. Se trata de una exposición del desarrollo gradual que muestra relaciones vaso comunicantes entre los diferentes estadios de evolución ${ }^{5}$ y que expresa, a nivel universitario, las exigencias y contradicciones propias de una sociedad industrializada tal como se presenta en occidente luego de la Segunda Guerra Mundial.

Por cierto, la nueva forma de concebir la universidad supone un conjunto de desafíos pedagógico-metodológicos tendientes a adecuar las prácticas universitarias a un nuevo escenario de diversidad intelectual, étnica, racial, cultural y económica de los alumnos que acceden a los estudios superiores. Por esta razón, no resulta extraño que en este nuevo contexto los autores preocupados por las cuestiones de metodología del aprendizaje y la enseñanza universitaria en general, progresivamente, hayan comenzado a centrar su atención en las estrategias psicológicas adecuadas para lograr el efectivo aprendizaje de los sujetos cognoscentes, teniendo en consideración el nuevo escenario universitario ${ }^{6}$.

En el contexto de la masificación de la educación superior, como exigencia de las sociedades industrializadas, tres, por lo menos, nos parecen ser las ideas que justifican los esfuerzos de adecuación estructural y estrategias pedagógicas dirigidas a los nuevos estudiantes. Primera: el nuevo escenario de acceso a la educación superior está caracterizado por la diversidad social, económica, étnica, racial y cultural de sus alumnos. Segunda, la modificación estructural del sistema universitario y la aplicación de nuevos métodos adecuados al proceso de enseñanza-aprendizaje han de propender no solo a asegurar un acceso universal a las universidades sino también garantizar un egreso masivo de las mismas. Tercero, los nuevos tiempos hacen necesario que los procesos de formación no se limiten simplemente al trasvasije teórico de información, sino que propendan de manera decidida hacia una formación competencial enfocada en el futuro laboral de los jóvenes universitarios, cuestión fundamental en las sociedades industrializadas de carácter capitalista.

\footnotetext{
5 En tal sentido escribe Trow que "se debe enfatizar que el movimiento de un sistema de élite hacia uno de educación masiva o desde uno de educación masiva hacia uno universal de educación superior, no supone necesariamente que las formas y tendencias de la fase o fases anteriores desaparecen o son transformadas. Por el contrario, la evidencia sugiere que en cada fase sobreviven ciertas instituciones y partes de las otras; mientras el sistema considerado como un todo incrementa el número de estudiantes y funciones en la siguiente etapa. De manera tal que sus novedosas, y más importantes, instituciones tienen las características de la nueva fase. Así, en un sistema masivo, las instituciones de élite no solo sobreviven sino florecen; y las funciones de élite continúan siendo ejecutadas dentro de las instituciones del sistema masivo (de igual manera tanto las instituciones del sistema de élite como las del sistema de acceso masivo sobreviven en el tránsito de los Estados Unidos hacia un sistema de acceso universal a la educación superior). Sin embargo, esta observación señala un problema característico de las fases mixtas del sistema: el problema surge de las tensiones inherentes a la coexistencia de formas de educación superior fundamentalmente basadas en principios diferentes y orientadas por diferentes tipos de funciones". Trow (1973) pp. 19-20.

6 Una breve revisión de la bibliografía al uso sobre esta materia da cuenta de esta tendencia. El propio Johnston ofrece una lista de autores y textos de referencia entre los que destacan Teaching for Quality Lerning at University de John Biggs; Student Learning and University Yeaching editado por Entwistle y Tomlinson; Engaging the Curriculo in Higher Education de Barnett y Coate, y A Handbook for Teaching and Learning in Higher Education: Enhancing Academia Practice de Fry y Marshall.
} 
Todas estas ideas han tenido un impacto profundo en la vida universitaria. De manera tal que en los últimos años hemos asistido a un conjunto de reformas estructurales y curriculares de los centros universitarios, junto a una revisión constante de las prácticas didácticas, pensando en el nuevo modelo. A la reflexión general le ha sucedido una más particular: la que cada una de las ciencias ha realizado a propósito de su propio proceso formativo. De esta manera, cada parcela del conocimiento universitario ha tratado de hacer suyas las ideas precedentes pero adaptándola a sus necesidades propias. El derecho, por cierto, no ha estado ajeno a este proceso. En lo que sigue mostraremos cómo estas ideas han sido recogidas y desarrolladas, especialmente en el ámbito anglosajón, por los teóricos del derecho.

\section{LA ENSEÑANZA DEL DERECHO EN EL PARADIGMA DE LA EDUCACIÓN UNIVERSITARIA MASIVA Y DE ACCESO UNIVERSAL}

Como señalamos unas líneas más arriba, en los paradigmas de la educación superior masiva o de acceso universal a la enseñanza universitaria hay por lo menos tres ideas que han de ser consideradas en todo el proceso de diseño curricular y didáctica universitaria: i.- la presencia en los cursos de inicio de diversos tipos de alumnos, respecto de los cuales el sistema universitario debe hacerse cargo; ii.- la exigencia institucional de un número aceptable de egresados universitarios como contraprestación a la inversión pública o privada en el proceso de formación de los mismos, y iii.- la propensión a una educación superior orientada preferentemente hacia la práctica profesional. Se trata de tres ideas conectadas entre sí que tienen un conjunto de virtudes prácticas, pero que también comportan un cúmulo de riesgos, como luego tendremos oportunidad de ver.

\subsection{LA DIVERSIDAD DE LOS ALUMNOS EN EL NUEVO CONTEXTO UNIVERSITARIO}

Los nuevos estudiantes universitarios son diversos de múltiples maneras. Son distintos social, cultural y económicamente. $\mathrm{Y}$ a estas diferencias habría que sumar otras: políticas, valóricas y religiosas. Y tal vez otras más, pues son también distintos sexual, racial, étnica y etariamente. En fin, los nuevos alumnos universitarios conforman un universo diverso con un propósito común: adquirir cierta instrucción intelectual que les permita desarrollarse como personas y mejorar sus propias condiciones de vida.

Aunque todas las diferencias antes mencionadas son importantes (sobre todo para el profesor), hay una que con mayor intensidad ha propiciado las restantes: la diversidad socioeconómica. De hecho, el hilo conductor de la tesis de Trow, sobre el cambio de paradigma de la educación superior, es precisamente la progresiva incorporación al mundo universitario de las clases sociales menos aventajadas. Se trata de un fenómeno característico de las sociedades industrializadas y políticamente democráticas de la segunda mitad del siglo XX.

Especialmente en los países de Europa Occidental y en los Estados Unidos de Norteamérica los cambios políticos y económicos acaecidos luego de la Segunda Guerra Mundial propiciaron el cambio de paradigma del modelo universitario de élite al de acceso masivo y luego al de acceso universal. Así, primero las clases medias y luego incluso las bajas vieron en el curso de unas pocas décadas cómo las instituciones universitarias (hasta hace poco 
reservadas solo para los ricos y los nobles) se volvían espacios asequibles para ellas. En este nuevo escenario no solo se jugaba una cuestión de movilidad social para las clases medias y bajas, sino algo más profundo e importante: la deconstrucción de los esquemas sociales tradicionales y la pérdida de los estatutos y privilegios asociados a dichos esquemas ${ }^{7}$.

Obviamente, estos cambios rápidamente exigieron otros de parte de las universidades. En primer lugar, de los esquemas de selección por mérito (sistema de élite) se pasó a los de igualdad de oportunidades (sistema de acceso masivo), para finalmente llegar a la supresión de los criterios de selección en los sistemas de acceso universal. En este último, el objetivo es alcanzar una igualdad en "los logros del grupo en lugar de una igualdad de oportunidades individuales; los esfuerzos se realizan para alcanzar a nivel social, de clase, étnico y racial una distribución de la educación superior que refleje a la sociedad en general. Por supuesto, cuanto más temprana sea la edad en que los miembros de la sociedad entren a este sistema, de forma más directa este reflejará la distribución en los subgrupos en la sociedad en general. En su grado último, por cierto (este sistema), es 'democrático', en el mismo sentido en que lo son las formas obligatorias de educación primaria y secundaria”.

En segundo lugar, y como consecuencia de lo anterior, las universidades se vieron en la necesidad de someter a examen sus prácticas de gobierno, programas académicos e incluso su propio rol en la sociedad. Esta revisión puso en evidencia la necesidad de acometer grandes reformas al sistema universitario, aunque respecto a la naturaleza y alcance de las mismas no existiera acuerdo dentro de la academia. Trow distingue diversas actitudes de los académicos frente a las reformas del sistema universitario. Por una parte, están aquellos que asumen una actitud "elitista tradicional", para quienes la universidad sigue siendo un espacio reservado para las élites intelectuales. Para este grupo, con todo, más importante que los sistemas de acceso a la educación superior son los criterios de egreso de los estudiantes. En otras palabras, la cuestión no es solo quién pude acceder a la educación universitaria sino quién tiene las aptitudes y competencias para egresar de un sistema altamente competitivo cuyo centro de actividad es solo la generación y promoción del conocimiento humano. En el otro extremo de los "elitistas" se encuentran los "reformadores expansionistas". Para estos, la universidad debe replicar el modelo político democrático y en tal sentido afirman que "debe haber una transformación sustancial de la educación superior; esta debe ser extendida verticalmente en la estructura de clases -fundamentalmente democratizada en sus patrones de reclutamiento- y también horizontalmente en una amplia gama de actividades sociales, económicas y políticas de la sociedad"'. Para los "reformadores expansionistas" mientras no se produzca una verdadera revolución de las estructuras tradicionales de la educación universitaria (en términos de mayor inclusión democrática en el gobierno de las mismas) las sociedades occidentales seguirán caracterizadas por la segregación social y la concentración del poder en manos de una minoría. Entre una y otra posición extrema están

El historiador británico Eric Hobsbawm ha señalado que este proceso de democratización de la educación superior supuso para las clases trabajadoras una suerte de "emancipación" de los esquemas culturales heredados y como tal una liberación de las relaciones de dependencia y subordinación de las clases dominantes. Véase a este respecto НовsваWм (2013) pp. 99-115.

8 Trow (1973) p. 14.

9 Trow (1973) p. 32. 
los denominados "expansionistas tradicionalistas" y los "reformadores elitistas". Los primeros aceptan las ideas de acceso masivo e incluso universal pero no la expansión de los valores tradicionalmente asociados a la universidad (búsqueda del conocimiento); los segundos, por el contrario, están abiertos a la posibilidad de que las universidades adquieran otros compromisos (no solo académicos); pero piensan que el gobierno y la gestión universitaria debe mantenerse en manos de una élite intelectual (el claustro académico), respecto de la cual las ideas democráticas no son aplicables.

Finalmente, el nuevo modelo de educación superior supuso una modificación en las prácticas pedagógicas y curriculares universitarias con el propósito de formar estudiantes muy diversos entre sí, sin renunciar a los estándares tradicionales de exigencia y excelencia universitaria. Así, en el sistema de acceso universal a la educación superior la cuestión sobre la "buena calidad de la educación" muy pronto se transformó en un verdadero problema. Por una parte, los costos de mantenimiento de este sistema son muy superiores a los de un esquema de educación universitaria de élite; y, por otra, los alumnos que en este modelo ingresan a la educación superior están menos preparados para adquirir una formación universitaria.

Los mayores costos del sistema inciden así en las inversiones que buenamente se pueden realizar en las diferentes universidades (a quiénes y qué áreas de investigación se fomentan); y las aptitudes y conductas de entrada de los alumnos, a su vez, repercuten en los resultados esperables del proceso. De tal forma que, siendo los recursos insuficientes y los alumnos mal preparados, el resultado ha de traducirse probablemente en un deficiente desempeño de los estudiantes universitarios. Y sin embargo, aunque lógicamente el resultado tiende a lo anterior, el sistema ha de buscar las formas internas de corrección dentro de un proceso irreversible. De ahí que cobren importancia en estos modelos las ideas de "tasas aceptables de retención"; "educación continua"; "especialización”; etc.

Así las cosas, el nuevo paradigma de acceso universal a la educación superior progresivamente llevó al cuestionamiento de los procesos de selección; de las formas tradicionales de gobierno y administración de las universidades; de los modelos de enseñanza y de los curricula, y de las estrategias de financiamiento del sistema. Todas estas cuestiones asociadas a la diversidad de los nuevos alumnos universitarios, como era de esperar, también afectaron a las escuelas de derecho.

En efecto, en el ámbito propio de la enseñanza y didáctica del derecho, el nuevo paradigma del acceso universal a la educación universitaria y su derivada natural: la mayor diversidad de los nuevos alumnos del sistema, es un tema que solo en los últimos años ha recibido la atención que merece; tal vez porque antes de ser un "tema" de discusión propiamente tal se lo ha dado por supuesto en todas las discusiones sobre la enseñanza jurídica ${ }^{10}$. De hecho, en los Estados Unidos de Norteamérica, la discusión sobre los beneficios y problemas derivados de la diversidad de los estudiantes ha cobrado real importancia solo a partir de 1996 a propósito del caso Hopwood.

\footnotetext{
10 En este sentido Gerald F. Hess ofrece una muy completa bibliografía con títulos que tratan prácticamente de todos los problemas asociados a la enseñanza y didáctica del derecho, pero nada dice respecto a la cuestión de la diversidad de los estudiantes en las escuelas de derecho. Véase Hess (2000) pp. 63-169.
} 
En "Diversidad y Educación Legal: Experiencia Estudiantil en Escuelas de Derecho Líderes", Gary Orfield y Dean Whitla examinan las diversas posiciones que los teóricos, los jueces y los alumnos de algunas de las más prestigiosas escuelas de derecho sostienen sobre este delicado tema. Como fondo de la discusión aparecen los fallos judiciales Regents of the University of California vs. Bakke ${ }^{11}$ de 1978 y Hopwood vs. Texas ${ }^{12}$ de 1996. El primero es considerado una ratificación jurisprudencial a las llamadas "acciones afirmativas" en el ámbito universitario; mientras que el segundo una señal de alerta y retroceso respecto a los avances en materia de integración.

Para Orfield y Whitla resulta indudable que la mayor diversidad del alumnado en el esquema actual de la educación superior de acceso universal constituye un beneficio. Y si esto es verdad respecto del sistema universitario en general es aún más evidente en el ámbito de la educación jurídica, pues "los valores afirmados por el juez Powell y por los funcionarios de admisión de Harvard, citados en el caso Bakke, parecen estar actuando hoy en las vidas de los estudiantes de derecho. Es criticable que el mundo académico haya sido lento en estudiar estos cambios. Sin embargo, estos datos claramente afirman los juicios hechos hace treinta y cinco años por las Cortes y de los líderes de la educación legal, cuando ellos se embarcaron en políticas que llevaron a la diversidad, que la mayoría de los estudiantes actuales encuentra tan beneficiosos para su educación legal y el entendimiento crítico de las diferentes dimensiones de su profesión"13.

Yendo más lejos aún, Kevin R. Johnson ha puesto de manifiesto no solo la importancia de la diversidad de los alumnos en las escuelas y facultades de derecho, sino también la de los académicos que forman parte de las mismas.

Tomando como punto de partida de su argumento el caso Grutter v. Bollinger ${ }^{14} \mathrm{de}$ 2003, Johnson afirma que la diversidad en sus diferentes manifestaciones (estudiantes y

11 El caso Bakke se inscribe dentro de la discusión norteamericana de las llamadas "acciones afirmativas". Estas constituyen una serie de medidas legales y administrativas tendientes a asegurar la inclusión social en diferentes ámbitos. En el caso de Allan Bakke, paradójicamente, las acciones afirmativas tomadas por la Universidad de California (consistentes en reservar el 16\% de los cupos de admisión para la Escuela de Medicina de Davis a grupos étnicos minoritarios) perjudicaban a Bakke, quien pese a tener un promedio de calificaciones superior a muchos de los seleccionados por pertenecer a grupos minoritarios, no obtenía un cupo. La estrategia de Bakke fue argumentar discriminación racial, pero a su favor. Finalmente la Corte, sin desconocer el valor de las acciones afirmativas, acogió la demanda de Bakke y ordenó su aceptación a la Escuela de Medicina de Davis; dejando en claro que para la realización de una acción afirmativa no basta el criterio de la "cuota"; sino que han de ponderarse otros factores para propender a la integración. Este criterio es el que ha seguido imponiéndose jurisprudencialmente en Estados Unidos. Para un análisis detallado del caso, véase Celis-Giraldo (2009) pp. 103-117.

12 La Corte del Distrito Oeste de Texas, conociendo de la negación de acceso a la Escuela de Derecho de la Universidad de Texas a la estudiante Cheryl J. Hopwood, por motivos similares a los del caso Bakke, estimó que no se debían usar acciones afirmativas en la universidad como remedio a la segregación racial y social. Según la Corte no había evidencia de que la pluralidad en el estudiantado favoreciera la inclusión social ni la convivencia universitaria. Aunque en un primer momento la Corte Suprema no quiso conocer de la apelación que la Universidad de Texas realizó en contra del fallo; en 2003 en el fallo Grutter versus Bollinger, confirma la jurisprudencia del caso Bakke y, aunque de forma moderada, valida la importancia de las acciones afirmativas.

13 Orfield y Kurlaender (2001) p. 172.

14 Al igual que en el caso Hopwood, a Barbara Grutter se le negó el acceso a la Escuela de Derecho de la Universidad de Michigan, aunque sus calificaciones eran superiores de las de algunos postulantes de minorías 
académicos) produce un beneficio global al sistema educativo, beneficio que ha conducido, por una parte, al desarrollo de una noción más comprensiva del concepto de "diversidad" y, por otra, a un reconocimiento gubernamental y judicial del valor intrínseco de la inclusión en todas las áreas de la educación legal; cuestión que se ha visto reflejada, en el modelo norteamericano, en el propio establecimiento de las acciones legales y en los fallos judiciales desde la década del 50 en adelante.

Desde la perspectiva de la diversidad, los alumnos de derecho se ven "mejor preparados para triunfar y desarrollarse en este mundo real increíblemente diverso de abogados y clientes que es el de los Estados Unidos y también el del mundo. Los educadores universitarios han hecho suya la justificación moderna dominante invocada por la acción afirmativa de que la diversidad racial y de otro tipo provee beneficios educacionales tangibles y concretos a los estudiantes" ${ }^{15}$. Pero, además, en el otro extremo, la diversidad de los académicos, también, provee puntos de vista y soluciones jurídicas que serían impensables sin su presencia. En tal sentido, "no es la sala de clase el único lugar en donde la diversidad de una facultad de derecho importa. Diferencias de perspectiva, experiencia y conocimiento pueden influenciar la vida académica tanto como ellas pueden afectar la enseñanza. Incluso si uno no se siente cómodo con la expresión "el color de la voz", es incuestionablemente verdadero que, tal como en la enseñanza, los miembros de diferentes grupos minoritarios en su conjunto aportan más experiencias de vida diferentes, perspectivas y conocimientos para el desarrollo del análisis del derecho y la doctrina legal que sus colegas blancos"16.

Johnson advierte, sin embargo, que en el sistema norteamericano, aun cuando existe una base gubernamental y judicial que va en la dirección correcta de las acciones afirmativas, las mediciones más prestigiosas sobre la excelencia de las escuelas de derecho no toman en consideración los elementos de inclusión a la hora de confeccionar los diferentes rankings. "En efecto, el U.S. News rankings metodológica y argumentadamente penaliza a las escuelas de derecho que buscan promover la diversidad. Un reporte del Comité Especial en EE.UU. sobre Nuevos Rankings para la Sección de Educación Legal y Admisión a la Barra de 2010 concluye que, en su evaluación de la calidad de una escuela de derecho, el presente News 'metodológicamente tiende a reducir los incentivos a la diversidad de la educación legal"17. Y esto claramente se opone al propio discurso público sobre el valor de la diversidad y a la idea del beneficio académico supuesto en la misma.

La experiencia norteamericana muestra, en todo caso, que la dificultad que nos habíamos formulado unas líneas más arriba (¿cómo conciliar la exigencia académica tradicional de las universidades con el ingreso masivo de alumnos diversos?) se desactiva cuando se comprende que en el nuevo paradigma de la educación superior de acceso universal los valores asociados a la actividad universitaria no se limitan a la generación y promoción del conocimiento sino a un conjunto de valores sociales que se relacionan con la inclusión y el

(afroamericanas e hispanas). En este caso, aunque el tribunal de primera instancia acogió la demanda de Gutter, primero la Corte de Apelaciones y luego la Corte Suprema, confirmaron la jurisprudencia del caso Bakke; en el sentido de reconocer un valor intrínseco a las acciones afirmativas; pero sin alusión al sistema de "cuotas".

15 Johnson (2011) p. 1552

16 JOHNSON (2011) p. 1562

17 Johnson (2011) p. 1576 
enriquecimiento de la perspectiva social desde la cual se produce el conocimiento mismo. Por cierto, esto no supone renunciar a una educación de calidad, pero sí revisar las prácticas y métodos pedagógicos al uso en el nuevo paradigma. Esto explica, a su vez, el incréble interés que existe por parte de los teóricos de la educación legal por desarrollar técnicas de enseñanza y evaluación congeniales con la nueva realidad ${ }^{18}$.

\subsection{LA EXIGENCIA INSTITUCIONAL DE UN NÚMERO ACEPTABLE DE ALUMNOS Y DE EGRESADOS UNIVERSITARIOS}

La educación superior de acceso universal es un fenómeno irreversible; y hay buenas razones para alegrarse de ello. Hemos visto cómo en el ámbito de la educación jurídica todo parece indicar que la diversidad social que se promueve en el nuevo paradigma universitario es percibida por los estudiantes y académicos de manera positiva. Ella, por una parte, brinda a los alumnos la posibilidad de comprender el mundo y actuar en él desde una perspectiva realista (vivimos en un mundo diverso). Por otra, la diversidad en la academia permite alcanzar nuevos horizontes en la reflexión jurídica.

A lo anterior hay que añadir que los estudios muestran, también, que hay un potencial beneficio en el nuevo modelo universitario: el incremento del capital humano social y su consecuente reflejo en el dominio de la economía de un país ${ }^{19}$.

Sin embargo, la educación universitaria masiva, más allá de sus beneficios, suponen una serie de desafíos económicos para cualquier país. El aumento del número de matrículas en las universidades, el incremento de las plazas de académicos y el mayor número de recursos físicos (edificios e insumos) que el sistema requiere han elevado a la categoría de problema fundamental la cuestión sobre el financiamiento del nuevo modelo universitario. Se trata de un problema relativamente nuevo, que no estaba presente en el sistema de la educación universitaria de élite por la sencilla razón de que en aquel el número de alumnos y académicos era considerablemente menor y los recursos propios de los educandos y sus exclusivas instituciones eran bastante mayores.

Pues bien, todo sistema universitario masivo o de acceso universal debe financiarse. En algunos casos el compromiso financiero lo adquiere el estado (sistemas universitarios públicos) y en otros los particulares. Pero, sea cual sea el sistema de financiamiento, siempre quien lo soporta tiene un interés comprometido en ello. Cuando el sistema es solventado por el erario el interés está en el bien social que produce la universidad (capital humano e incremento económico nacional); cuando quien paga es el particular, el interés está, en el mejor de los casos, en financiar la propia universidad.

La cuestión del financiamiento del sistema universitario, a su vez, se conecta indisolublemente con otra: el problema de las "tasas de retención y graduados", esto es, la capacidad que tiene una universidad para mantener dentro del sistema al mayor número

18 Para una bibliografía bastante completa sobre este punto se puede consultar KoCHAN (2011) pp. 449-471.

19 A este respecto véase Educational at Glance 2014 OCDE Indicators, especialmente el capítulo A. Disponible en http://www.keepeek.com/Digital-Asset-Management/oecd/education/education-at-a-glance-2014_eag2014en\#page7. De acuerdo a la OCDE existe una directa relación entre el nivel de escolaridad de la población y el desarrollo económico de la misma. Asimismo, a mayor incremento en el número de personas que acceden a la educación (en todos sus niveles) mayores son los índices de participación e igualdad en la sociedad. 
posible de sus alumnos y, en un plazo razonable, convertirlos en graduados. Las tasas de retención y egreso son importantes económicamente tanto para quien financia el sistema universitario como para los usuarios del mismo (alumnos y padres de los alumnos). Si quien financia el sistema universitario es el Estado, los índices de retención y egreso son vistos como la contraprestación a la inversión pública con miras al beneficio social que supone el incremento del capital humano. Si es un particular quien financia la universidad, las tasas de retención y egreso constituyen un medio de subsistencia de la institución universitaria dentro del sistema. Para los alumnos y sus padres (como también para los gobiernos), las tasas de retención y egreso funcionan como indicador de la calidad de la educación que han de recibir los universitarios y como garantía de que el tiempo y recursos invertidos en el proceso formativo culminará con un grado académico que mejorará sus perspectivas de desarrollo económico y social ${ }^{20}$. Como se ve, más allá de que el sistema de acceso universal sea a universidades públicas, privadas o mixtas, siempre la cuestión de las tasas de retención y egreso, en el nuevo paradigma, será un tema fundamental.

Pero, aun cuando todo sistema de educación superior masivo o de acceso universal requiera de financiamiento, y este último conduzca a la cuestión de las tasas de retención y egreso, la justificación de estos índices varía significativamente desde el modelo de financiamiento público al privado. En el modelo público la razón de ser de la tasa de retención y egreso es el incremento en el capital humano social que se espera como contraprestación a la inversión pública. En esta misma dirección, los incentivos por captación, retención y egreso de alumnos obedecen más bien a necesidades sociales y no de mercado. En el modelo de financiamiento privado, por el contrario, la cuestión de las tasas de retención y egreso puede responder, además del incremento del capital humano, a cuestiones de mercado y, en última instancia, a un problema de supervivencia de la propia institución universitaria. Así, aunque para todo sistema de educación superior masiva o de acceso universal la cuestión de las tasas de retención y egreso es fundamental, la razón de ser de las mismas varía considerablemente dependiendo de si estamos dentro de un sistema de educación universitaria de financiamiento público o privado ${ }^{21}$.

El sistema de educación superior alemán constituye un buen ejemplo del modelo de educación universitaria pública y, por lo mismo, en aquel la cuestión de las tasas de retención y egreso está indisolublemente ligada a las necesidades sociales de incremento de capital humano en áreas específicas. Esta vinculación entre el incremento de la retención estudiantil, el número de graduados y las necesidades sociales se refleja en las Universidades e Instituciones Técnicas de Nivel Superior (Fachhochschulen) alemanas en diversos aspectos. En primer lugar, el sistema alemán distingue entre carreras de número cerrado y carreras de

\footnotetext{
20 En los Estados Unidos de Norteamérica, bastante liberal en lo que a educación universitaria toca, este es un tema que se encuentra regulado legalmente. "La Ley Federal sobre Derecho a la Información de los Estudiantes y Seguridad de Campus de 1991 representa un intento sin precedentes por el gobierno federal para requerir de las instituciones de educación superior la publicidad de sus datos sobre la 'calidad' de sus programas educacionales. Entre otras cosas, esta ley requiere de las instituciones hacer pública la información sobre finalización (de estudios) o tasas de graduados". AusTin (1997) p. 647.

21 Para un análisis detallado sobre los diferentes modelos universitarios a propósito de este y otros temas, véase Salaburo (2003).
} 
número abierto. Las primeras tienen un número limitado de matrículas en razón de una saturación de mercado; las segundas no. Mediante esta estrategia se busca evitar que exista un número superior de graduados al necesario en una determinada área del conocimiento. En segundo lugar, los requisitos de titulación, especialmente para la carrera de derecho, constituyen otro elemento que incide en los niveles de retención y, sobre todo, de término satisfactorio de los estudios universitarios en Alemania (luego de completar sus estudios universitarios de forma satisfactoria en una facultad de derecho, el estudiante que quiera ser promovido a la calidad de abogado ha de rendir el Erste Juristische Prüfung-Referendarexamen- y luego el Zweite Juristische Prüfung-Assessorexamen-). Finalmente, la cuestión del financiamiento público de las universidades se resuelve en el caso alemán por criterios de oferta académica (vinculada a las necesidades sociales) y nunca por consideraciones de demanda de servicios educativos ${ }^{22}$, cuestión que hace que los temas de financiamiento universitario y retención, aunque vinculados, se mantengan debidamente diferenciados.

De esta manera en el sistema universitario alemán la cuestión de las tasas de retención y graduados de las universidades se encuentra fuertemente influenciado por las propias condiciones de un modelo que permite justificar racionalmente la inversión pública (financiamiento) que se realiza en las universidades, sin sacrificar las necesidades sociales ni los compromisos de excelencia académica tradicionales en ese país ${ }^{23}$.

En el otro extremo se encuentran algunas universidades de los Estados Unidos de Norteamérica. El sistema norteamericano no es, por cierto, totalmente privado, sino más bien uno que combina instituciones de carácter público con otras de naturaleza privada con y sin fines de lucro. Sin embargo, a fin de mostrar el contraste con el sistema de educación superior pública, limitaremos nuestro análisis a las necesidades de retención de las instituciones cuyo financiamiento es privado y tienen fines de lucro. En los Estados Unidos estas universidades son llamadas "proprietary universities" y representan un porcentaje mínimo dentro del sistema: aproximadamente el 17\%.

Las universidades privadas con fines comerciales nacieron en los Estados Unidos de América a finales del siglo XIX con una vocación práctica. Se trataba de pequeñas escuelas de oficios (con cursos de secretariados o contabilidad básica) que ofrecían a sus estudiantes la posibilidad de recibir una formación técnica, de corto tiempo, que les permitiera mejorar

22 Para un estudio detallado sobre las fuentes de financiamiento del sistema alemán y europeo en general, véase Salaburo (2007) pp. 407 y ss.

23 Sin perjuicio de lo anterior, los teóricos del derecho alemán no han descuidado los aspectos que tienen que ver con la adaptación de las estrategias de enseñanza aprendizaje en el nuevo escenario universitario. En tal dirección, merece una especial mención los esfuerzos que durante los últimos años ha realizado el Centro para la Didáctica de las Ciencias del Derecho de la Universidad de Hamburgo. Dichos esfuerzos han quedado plasmados en una serie de libros que no han descuidado ninguno de los aspectos de didáctica, planificación y evaluación universitaria. Véase en tal sentido: Brockman et al. (2011); Brockman y Dietrich (2012); Griebel y Gröblinghoff (2012); Brockman y Pilniok (2013), y Brockman \& Pilniok (2014). De alguna manera, aunque las facultades de derecho alemanas han opuesto una fuerte resistencia a los cambios que demanda la adaptación al Espacio Europeo de Educación Superior, no se puede descartar que los nuevos esfuerzos didácticos y de modificación curricular llevados a cabo en los últimos años en las escuelas de derecho alemanas, en parte, han sido motivados por cuestiones de índole política más que académica. A este respecto, Chistian Baldus ha mostrado con claridad la influencia que sobre esta materia ha ejercido el informe del Wissenschaftrat. Véase sobre el particular Baldus (2013) pp. 5-58. 
sus expectativas laborales. En un comienzo estas instituciones tuvieron bastante éxito, pues no era mucho lo que se debía invertir en ellas (profesores e infraestructura) y, por lo general, sus graduados lograban sus objetivos y, por lo mismo estaban dispuestos a sufragar ellos mismos los gastos de su educación.

En la actualidad su fuente de financiamiento y objetivos siguen siendo los mismos (obtienen sus ingresos de las matrículas y sus actividad se circunscribe al ámbito de la docencia y no al de la investigación), aunque algunas de ellas han aumentado considerablemente sus fuentes de financiamiento y objetivos, llegando incluso a cotizar en la bolsa de valores de Nueva York (este es el caso, por ejemplo, de la University of Phoenix; de la University of Arizona, o del De Vries Institute de Illinois). Estas propietary universities funcionan en la misma lógica del mercado de los bienes de consumo. En tal sentido, "las universidades con ánimo de lucro dependen de la venta directa de oferta de cursos y la comisión ( $b$ board») que las gobierna requiere la obtención de beneficios sobre las inversiones realizadas, como sucedería con cualquier otro tipo de empresa" ${ }^{24}$.

Desde una perspectiva muy congenial con las propietary universities, Ormond Simpson ha criticado la falta de sensibilidad y estrategia de algunas universidades para advertir los riesgos económicos que suponen los bajos índices de retención. Simpson advierte que "la educación superior es un extraño negocio. Ninguna otra forma de industria podría tomar a prueba sus componentes (estudiantes nuevos) y producir un producto final (graduados) con un desperdició del $20 \%$ o más. En último término, si tal negocio existiera este muy rápidamente iría a quiebra. Aun así, las universidades no solo ignoran tal desperdicio sino parecen tener un orgullo perverso en ello, pues mantenerlos supone un alto estándar académico" 25 . Obviamente esta crítica es válida en la medida en que se considere la universidad como una empresa y a su actividad como una actividad económica. Cuestión discutible.

Como se observa, en el caso específico de las propietary universities las ideas de financiamiento universitario y tasas de retención y término de los estudios superiores se encuentran vinculadas de manera más intensa que en el sistema universitario alemán. Y esto es un elemento perturbador, toda vez que puede conducir a la promoción irracional de oferta académica sin una base real de exigencia social, específicamente tratándose de carreras que no requieren para su implementación de grandes inversiones (derecho entre ellas). En el sistema de los Estados Unidos, con todo, esto no es un problema si se considera el número prácticamente insignificante de estas instituciones dentro del sistema en su conjunto y los requisitos para obtener el título de abogado.

Ahora bien, sea que las tasas de retención y graduados justifiquen la inversión pública por el aumento de capital humano o la inversión privada por la subsistencia de la propia universidad, en uno y otro caso existe la convicción común de que el aumento de la escolaridad superior de la población incide de manera directa en el incremento económico de la sociedad. Se trata de una convicción que tiene una base racional, como lo demuestran los últimos estudios de la OCDE (Educational al Glance 2014 OCDE Indicators) y que se conecta con una suposición tradicional sobre los beneficios individuales de la formación

\footnotetext{
4 Salaburo (2003) p. 161.

25 SimpSON (2005) p. 34.
} 
universitaria: la promoción social de los individuos de las clases menos aventajadas de la sociedad mediante este tipo de educación.

Sin embargo, la relación necesaria que se ha establecido entre "promoción social" mediante la educación universitaria y aumento de las riquezas económicas a causa de la misma, hoy está en tela de juicio. La razón es bastante simple: en el sistema de acceso universal a la educación superior existe un fuerte incremento del número de alumnos que accede a este tipo de formación, aumentando también significativamente el número de graduados universitarios disponibles en el mercado laboral. Debido a las leyes de la oferta y la demanda, el aumento de graduados influye tanto en su empleabilidad como en el monto de las remuneraciones que han de percibir gracias a sus estudios. "Como explica Scott 'los viejos vínculos entre la educación superior y las ocupaciones de élite de forma inevitable e inexorable se encuentran diluidas en los sistemas masivos. Esos nexos aún existen, por cierto. Las universidades mantienen el monopolio en la producción de doctores y abogados. Pero muchos graduados de los sistemas masivos no ocuparán tales roles privilegiados y prestigiosos en el mercado laboral. De hecho, llenarán los puestos de los mandos medios y los cargos de la burocracia pública, siempre en expansión”26.

En todo caso, aun cuando las expectativas laborales de los nuevos graduados probablemente no se vean del todo satisfechas en las actuales condiciones del mercado, sigue siendo cierto que la masificación de la educación trae un considerable número de beneficios económicos, sanitarios y políticos para la sociedad en su conjunto ${ }^{27}$. Eso sí, el mantenimiento de estos beneficios en el tiempo supone un compromiso público serio por regular las condiciones en las que se desarrolla el sistema de educación superior.

\subsection{LA PROPENSIÓN A UNA EDUCACIÓN JURÍDICA ORIENTADA PREFERENTEMENTE HACIA LA PRÁCTICA PROFESIONAL}

Las universidades de acceso universal no solo se distinguen de las antiguas universidades de élites en cuanto a su composición (diversidad de sus alumnos y académicos), sino también en cuanto a sus fines y cometidos. Las actuales universidades occidentales no solo buscan la producción y promoción del conocimiento, sino también la promoción social, el mejoramiento de las condiciones de vida de la comunidad y el fomento de la conciencia cívica (en la mayoría de los casos vinculada a las ideas rectoras de los regímenes democráticos). En el nuevo paradigma universitario existe también, y por lo mismo, mayor conciencia sobre la importancia práctica de la formación académica, cuestión que ha llevado de manera natural a las universidades a hacerse cargo no solo de entregar una instrucción teórica a sus alumnos sino también un conjunto de competencias y destrezas que les permitan desenvolverse de manera adecuada en el ámbito social y laboral.

\footnotetext{
26 JOHNSON y LEWIS (2005) p. 359

27 Un interesante estudio sobre el impacto que tiene el nivel de escolaridad en los ámbitos económicos, sanitarios y políticos se puede encontrar en Hannun \& BUCHMAN (2005) pp. 333-354. Aunque no se trata de una investigación que esté circunscrita al ámbito propio de la educación universitaria, sí es muy ilustrativa de los mitos y realidades sobre el real impacto de la educación en los países desarrollados y en vías de desarrollo.
} 
En el caso específico de la enseñanza del derecho, la reflexión sobre la necesidad de una formación jurídica no solo teórica sino también práctica es anterior al cambio de paradigma universitario ${ }^{28}$. Sin embargo, solo a partir de los últimos años se ha precisado con mayor claridad qué ha de entenderse por "habilidades jurídicas" y cómo estas han de ser idealmente integradas en el currículo.

En el caso norteamericano, el Report of the Task Force on Law Schools and the Profession: Narrowing the Gap de $1992^{29}$ dedica su capítulo tercero sección B "a una mirada de conjunto a las habilidades y valores analizados". Se trata de un conjunto de habilidades cognitivas (solución de problemas, razonamiento legal, investigación teórica y empírica) y prácticas (comunicación, capacidad de consejo y negociación, habilidades para litigar, organizar grupos y resolver dilemas éticos) y valores profesionales (competencia profesional, compromiso con la profesión y la justicia) que la Comisión Especial ha indicado como fundamentales en la formación de los futuros abogados, además de los conocimientos jurídicos sustantivos que han de adquirir.

Todas estas competencias y valores tienen por objetivo establecer un vínculo más estrecho entre la teoría y la práctica del derecho. En efecto, "cuando la Comisión Especial comenzó a considerar cómo la preparación de los abogados para la práctica podía ser mejorada, sintió la necesidad de desarrollar una concepción del objeto de esta preparación, en la forma de un compendio de habilidades y valores que deseablemente han de tener quienes practican la profesión" ${ }^{30}$.

Las ideas de la Comisión Especial, de acuerdo al propio informe, no constituían una imposición para las escuelas de derecho, sino más bien una suerte de carta de navegación que les indicaba el objetivo a alcanzar mediante las respectivas reformas curriculares. Sin embargo, diez años después del informe, la situación en las escuelas de derecho de los EE.UU. no evidenciaba la reforma estructural de los curricula que los miembros de la Comisión esperaban. En tal sentido, Alice M. Noble-Allgire ha señalado con acierto que uno de los mayores problemas en la implementación de las ideas del MacCrate Report reside en la separación, dentro de las mallas curriculares de los programas de derecho, entre los cursos de "dogmática jurídica tradicional" y aquellos cursos denominados de "habilidades blandas". Los primeros tradicionalmente asociados a las habilidades cognitivas y los segundos a las habilidades prácticas y a los valores jurídicos. De este modo, "la integración de

\footnotetext{
28 Por ejemplo, en 1667 Gottfried Wilhelm Leibniz publicó De Nova Metodo Discendae Docendaeque Jurisprudentia. Se trata, en términos muy generales, de una obra de didáctica jurídica dividida en dos partes. La primera de ellas está consagrada a la determinación de los diversos ámbitos del conocimiento humano y de las bases psicológicas supuestas en el acto mismo de conocer. La segunda parte, a su vez, está destinada al análisis de lo jurídico en particular, sus bases filosóficas y la forma en que ha de organizarse su estudio, curricularmente hablando. Lo interesante del método propuesto por Leibniz reside, entre otras cosas, en su idea de incorporar al estudio del derecho el análisis de casos prácticos, y así evitar el academicismo escolástico en el que estaban atrapadas las facultades de derecho de su época. Para comprender la influencia que ejercieron estas ideas de Leibniz, incluso sobre el sistema norteamericano de enseñanza del derecho, véase HoEfich (1986) pp. 95-121.

29 Legal Education and Professional Development - An Educational Continuum. Report of the Task Force on Law Schools and the Profession: Narrowing the Gap, American Bar Association, Chicago, 1992.pp. 416. También citado como "MacCrate Report".

30 Legal Education and Professional Development - An Educational Continuum (1992) p. 123.
} 
habilidades y doctrina es controversial porque desafía lo que algunos en la academia perciben como una dicotomía entre sustancia/habilidades. La etiqueta de 'sustantivo' es aplicada a los cursos doctrinales que han sido durante mucho tiempo parte del currículo de la escuela de derecho, mientras que la etiqueta 'habilidades' se reserva para cursos enfocados en habilidades prácticas que alguna vez se esperó que los nuevos abogados aprendieran en el trabajo. Desafortunadamente, existe también una percepción de que los últimos cursos y habilidades son menos importantes para el currículo de una escuela de derecho" ${ }^{31}$.

Ahora bien, aun cuando la integración curricular de las habilidades y valores a los cursos tradicionales de dogmática jurídica sea resistida, es un hecho que la tendencia es ir progresivamente incorporándolas a los cursos tradicionales por las ventajas que esto representa. Para Noble-Allgire, las ventajas de la integración curricular se ven reflejadas en tres aspectos: por una parte los alumnos mejoran sus capacidades de aprendizaje, además, los cursos teóricos o doctrinales son comprendidos desde una perspectiva significativa para los alumnos y, finalmente, se produce una suerte de sinergia entre la práctica y la teoría, pues la combinación de las habilidades con el derecho sustantivo "permite a los estudiantes pensar holísticamente, más que en forma compartimentada. Los ejercicios para desarrollar las habilidades permiten a los estudiantes ver cómo el conocimiento sustantivo es aplicado en la práctica actual del derecho. Más importante, esos ejercicios ayudan a los estudiantes a reconocer las relaciones de interdependencia entre las diversas habilidades y valores" ${ }^{\prime 2}$.

Pero, no solo los curricula de las escuelas de derecho no han adecuado del todo sus mallas a las recomendaciones de la Comisión Especial, sino además, como han advertido Hricik y Salzmann, en los últimos 20 años se ha producido una progresiva separación entre la actividad académica realizada por los profesores de derecho y la actividad profesional. Esta situación se ve reflejada en el escaso impacto que tiene el trabajo legal científico en el ámbito del ejercicio de la profesión, de forma tal que "a diferencia de apoyarse en los artículos de revistas de derecho, los abogados litigantes están volviendo al derecho mismo como fuente de su razonamiento legal. Abogados y jueces, así, tienden a apoyarse en sus propias habilidades lógicas, más que a aplicar teorías legales sintetizadas por otros"33.

Esta situación tiene, por cierto muchas explicaciones: el grado extremo de abstracción que tiene los problemas que tratan los académicos en sus artículos; la desconexión de tales temas con los asuntos prácticos que interesan a la comunidad profesional; la falta de tiempo que afecta a abogados y jueces y que les impide leer con atención los trabajos científicos; la creciente exclusión en la producción científica del derecho de abogados litigantes y jueces, etc. En los hechos esto se ha traducido, en que "menos del tres por ciento de los recursos citados por la Corte Suprema han sido artículos de revistas de derecho, que se supone analizan la vanguardia de los asuntos legales, doctrina y teoría"34.

Sin embargo, esta es una situación que es menester revertir a la brevedad, pues "los académicos son los intermediarios entre la teoría legal de punta y aquellos que actualmente

\footnotetext{
31 Noble-Allgire (2002) p. 35.

32 Noble-Allgire (2002) p. 39.

33 HriciK y Salzman (2005) p. 768.

34 Hricik y Salzman (2005) p. 778.
} 
practican el derecho. No debemos denigrar el rol que la teoría tiene en dar forma al derecho. La práctica deja hoy poco tiempo a los abogados que ejercen o a los jueces para producir una nueva o profunda teoría legal. Solo los académicos tienen el tiempo y los recursos para generar nuevas ideas sobre el derecho o investigar cómo la teoría legal afecta e impacta a la población considerada como un todo" ${ }^{35}$. Así las cosas, aunque en el sistema universitario de los EE.UU. hace ya varios años se ha detectado, por una parte, la necesidad de una vinculación más profunda y eficiente entre los planes de educación de las escuelas de derecho y las habilidades profesionales, y, por otra, lo deseable que resultaría que la actividad de los académicos de derecho se tradujera en una influencia real en el ejercicio del mismo, aún se trata de una tarea pendiente.

Como hemos visto hasta el momento, el número y diversidad de los nuevos alumnos universitarios, las exigencias de tasas aceptables de retención-egreso y la necesidad de vincular de manera más eficiente la academia con el mundo laboral son características propias del nuevo modelo universitario de acceso universal. Por cierto no son las únicas, pero sí, a nuestro parecer, las más importantes. De ellas también se siguen una constelación de oportunidades, desafíos y riesgos que las universidades occidentales (cada vez más influenciadas por el modelo norteamericano de educación superior) no deben dejar de considerar. En lo que sigue examinaremos, brevemente, alguna de ellas, especialmente, en lo que a la enseñanza del derecho toca.

\section{CONSIDERACIONES FINALES: ALCANCES DE LA IMPLEMENTACIÓN DEL NUEVO MODELO UNIVERSITARIO DE ACCESO UNIVERSAL PARA LA ENSEÑANZA DEL DERECHO}

No es difícil advertir en el nuevo modelo de educación superior una serie de oportunidades para la enseñanza del derecho. El aumento en número y diversidad de los nuevos estudiantes puede contribuir a generar espacios de inclusión y movilidad social y, a la vez, propiciar un clima de juiciosa tolerancia frente a la diferencia. La promoción de profesionales de primera generación, a su vez, puede fomentar un incremento del capital humano, tanto individual como colectivamente. La presión que actualmente ejerce el nuevo modelo universitario sobre las escuelas de derecho, en orden a mantener tasas razonables de retención y egreso, puede servir como garantía de que la inversión en tiempo y dinero que realiza tanto el Estado como las familias ha de ser razonablemente retribuida en el tiempo. Finalmente, la necesidad cada vez mayor de establecer puentes reales entre la academia y el mundo profesional es una oportunidad para orientar la práctica académica a las necesidades laborales de los nuevos tiempos (ya no basta con que el abogado sea un "sabedor del derecho") y, también, para que la práctica profesional pueda nutrirse de nuevas ideas para enfrentar sus problemas.

Para que todas las oportunidades antes mencionadas puedan realizarse, las escuelas de derecho han de propender a modificar tanto su estructura y funcionamiento como los curricula. En tal dirección, habrán de diseñar nuevos programas académicos que, sin renun-

35 HriciK y Salzman (2005) p. 777. 
ciar a los clásicos contenidos de dogmática jurídica, den cabida a nuevas metodologías de la enseñanza del derecho, junto con introducir cursos de habilidades blandas. Deberán fortalecer, también, una estructura interna de carácter democrático que dé cabida a los nuevos estudiantes (y profesores) para construir colaborativamente espacios de enriquecimiento intelectual. Tendrán, además, que resolver la delicada cuestión de conciliar las expectativas de las autoridades, los alumnos y sus familias con las exigencias de una formación universitaria de alto nivel. Todos estos son algunos de los desafíos que enfrentan las escuelas de derecho dentro del nuevo paradigma universitario.

Sin embargo, en el nuevo modelo de educación superior, el éxito de las escuelas de derecho no solo depende de su capacidad para detectar las oportunidades y desafíos, sino también, y de forma más radical, de su aptitud para evitar los riesgos inherentes a las nuevas circunstancias del quehacer universitario. El creciente incremento en número y diversidad de los alumnos de las escuelas de derecho, unido a las tensiones que sobre ellas se ejerce, a propósito de las tasas de retención y egreso, fácilmente las puede conducir a renunciar a estándares mínimos de excelencia académica. Una oferta desmedida e irreflexiva de la matrícula en las escuelas de derecho también puede conducir, a mediano plazo, a una saturación del mercado laboral y a la consecuente falta de trabajo de los futuros graduados. Finalmente, la progresiva incorporación de cursos de habilidades profesionales orientados a preparar de mejor manera a los futuros abogados puede conducir a una tecnificación de la enseñanza del derecho y, en el peor de los casos, a una captura por parte del mercado de las propias escuelas.

Aunque para la elaboración de este ensayo hemos recurrido preferentemente a literatura anglosajona, es fácil advertir que las oportunidades, los desafíos y riesgos propios del sistema universitario norteamericano son aplicables también a nuestras universidades y escuelas de derecho. Por lo mismo, en estos tiempos de reforma puede ser de utilidad saber qué podemos esperar, para bien o para mal, de este nuevo paradigma universitario de acceso universal.

\section{BIBLIOGRAFÍA CITADA}

Abramovich, Víctor (1999): "La enseñanza del derecho en las Clínicas Legales de Interés Público", Cuadernos de Análisis Jurídico Universidad Diego Portales, Serie Publicaciones Especiales, No 9: pp. 61-94.

Agudelo, Martín (2000): "Hacia un Procesamiento Adecuado de la Justicia: una Propuesta de Enseñanza del Derecho Procesal para la Sociedad del Nuevo Milenio”, Revista de Derecho Universidad Central, No 1: pp. 69-94.

Alvear, Julio (2002): "Algunas Anotaciones sobre la Enseñanza de la Ética Jurídica”, Actualidad Jurídica, No 6: pp. 27-34.

AmunÁtegui, Carlos (2007): "La enseñanza del Derecho Romano a través de Gayo. Una experiencia docente", Ius Publicum, N¹8: pp. 13-22.

ArósticA, Iván (2009): "Formando Abogados Analistas", Actualidad Jurídica, No 19, Tomo II: pp. 579-584. 
Austin, Alexander (1997): "How 'Good' is your Institution's Retention Rates", Research in Higher Education, vol. 38, No. 6: pp. 647-658.

Baldus, Christian (2013): "Perspectiva de la Ciencia Jurídica en Alemania. A Propósito del Informe del Wissenschaftrat", Anuario de Derecho Civil, Tomo LXVI, fac. I.: pp. 5-58.

BEnfeld, Johann (2016): "La discusión sobre la enseñanza del derecho en Chile dentro del nuevo paradigma universitario: una tarea pendiente", Revista de Derecho Universidad Católica del Norte, vol. 23, No 1: pp. 143-171.

Brockman, Judith, Dietrich, Jan-Hendrik y Pilniok, Arne (2011): Exzellente Lehre im Juristischen, en BROCKMAN, Judith (Hrsg.), Schriften zur rechtswissenchaftichen Didaktik, vol. 1. (Baden-Baden, Nomos).

Brockman, Judith, Dietrich, Jan-Hendrik y Pilniok, Arne (2012): Methoden des Lernens in der Rechtswissenchaft, en BROCKMAN, Judith et al (Hrsg.), Schriften zur rechtswissenchaftlichen Didaktik, vol. 3. (Baden-Baden, Nomos).

Brockman Judith y Pilniok, Arne (2013): Prüfung in der Rechtswissenschaft. En Brockman Judith y Pilniok, Arne (Hrsg.), Schriften zur rechtswissenchaftlichen Didaktik, Vol. 4. (Baden-Baden, Nomos).

Brockman, Judith y Pilniok, Arne (2014): Studieneingangsphase in der Rechtswissenschaft, en Brockman Judith y Pilniok, Arne (Hrsg.), Schriften zur rechtswissenchaftlichen Didaktik, Vol. 5. (Baden-Baden, Nomos).

Caballero, Juan (2000): "La Orientación Humanista en el estudiante de Derecho", Revista de Derecho Universidad Central, No 1: pp. 43-48.

Calvinho, Gustavo (2009): "La enseñanza del derecho procesal en el siglo XXI", Revista de Derecho Universidad Católica de la Santísima Concepción, vol. 2, No 20: pp. 11-34.

Campusano, Raúl (2002): "La Enseñanza del Derecho Informático en Chile. Apuntes para una Respuesta”, Actualidad Jurídica, No 5: pp. 305-316.

Caprile, Bruno (2000): "La Enseñanza del Derecho: ¿Clase magistral o método activo?”, Actualidad Jurídica, No 2: pp. 127-131.

CÁrcamo, Juan (2002): "Cooperación a la Justicia como Aspecto Formativo Fundamental del Estudiante de Derecho", Revista de Derecho Universidad Central, No 3: pp. 17-28.

CATTÁn, Ángela (2002): "Algunas notas para una Nueva Metodología para la Enseñanza del Derecho Romano", Actualidad Jurídica, No 5: pp. 317-344.

Celis-Giraldo, Jorge Enrique (2009): "Las acciones afirmativas en educación superior: el caso de los Estados Unidos", Educación y Educadores, vol. 12, No 2: pp. 103-117.

Coloma, Rodrigo (2006): "Hacia una enseñanza del Derecho Efectiva", Revista Escuela de Derecho, año 7, № 7: pp. 123-141.

Coloma, Rodrigo (2005): "El ocaso del profesor Binns. Un ensayo acerca de la enseñanza del derecho en Chile", Revista Ius et Praxis, año 11, No 1: pp. 133-172.

Coloma, Rodrigo y Agüero, Claudio (2012): "Los Abogados y las Palabras. Una propuesta para Fortalecer Competencias Iniciales en los Estudiantes de Derecho", Revista de Derecho Universidad Católica del Norte, año 19, No 1: pp. 39-69.

Devoto, Carolina (2012): "El Método de Resolución de Casos en la Enseñanza del Derecho", Actualidad Jurídica, No 25: pp. 503-512. 
Devoto, Carolina (2014): "Hacia una Caracterización teórica del Docente Universitario en la Carrera de Derecho", Actualidad Jurídica, No 30: pp. 507-518.

EsCANDÓn, Jesús (2006): "Inquietudes persistentes respecto a la enseñanza de la Filosofía del Derecho", Sociedad Chilena de Filosofía Jurídica Social Anuario de Filosofía Jurídica y Social, No 24: pp. 109-120.

Espinoza, Óscar (2005): "Privatización y Comercialización de la Educación Superior en Chile: Una Visión Crítica”, Revista de Educación Superior, vol. XXXIV, 3, No 135: pp. 41-60.

Griebel, Jörn y Gröbilnghoff, Florian (2012): Von der juristischen Lehre, en Griebel, Jörn y Gröblinghoff, Florian (Hrsg.), Schriften zur rechtswissenchaftlichen Didaktik, vol. 2. (Baden-Baden, Nomos).

Guzmán, Alejandro (2006): "La Enseñanza del Derecho. Historia y Perspectivas" en Anales del Instituto de Chile (Santiago, Instituto de Chile) pp. 272-406.

Hannum, Emily y Buchman, Claudia (2005): "Global Educational Expansion and SocioEconomic Development: An Assessment of Findings from the Social Sciences”, World Development, vol. 33, No. 3: pp. 333-354.

Hess, Gerald F. (2000): "Monographs on Teaching and Learning for Legal Educators", Gonzaga Law Review, vol. 35, special edition: pp. 63-169.

Hobsbawm, Eric (2013): Un Tiempo de Rupturas: Sociedad y Cultura en el Siglo XX (Trad. Cecilia Belza y Gonzalo García. Buenos Aires, Crítica, primera edición).

Hoeflich, Michael (1986): "Law and Geometry: Legal Science from Leibniz to Langdell", The American Journal of Legal History, vol. 30, No 2: pp. 95-121.

Hricik, David y Salzman, Victoria S. (2005): "Why There Should Be Fewer Articles Like This One: Law Professors Should Write More for Legal Decision-Makers and Less for Themselves", Suffolk University Law Review, vol. XXXVIII: pp. 761-787.

Jaeger, Werner (2010): Paideia: los Ideales de la Cultura Griega (Trad. Joaquin Xirau y Wenceslao Roces. Ciudad de México, Fondo de Cultura Económica, vigésimo primera edición).

JiMÉNEZ, Manuel (2011): "La movilidad académica como factor auxiliar del aprendizaje del Derecho”, Revista de Derecho, Universidad Católica de la Santísima Concepción, vol. 2, No 24: pp. 13-22.

Johnson, Kevin (2011): "The Importante of Student and Faculty Diversity in Law School: One Dean's Perspective”, Iowa Law Review, vol. 96-5: pp. 1549-1577.

Johnson, Bill (2010): The First Year at university. Teaching Students in Transition (Glasgow, McGrau-Hill, primera edición).

Johnson, Brenda y LewIS, Elton (2005): "German and UK Higher Education and Graduate Employment: The Interface between SystemicTradition and Graduate Views", Comparative Education, vol. 41, No. 3: pp. 351-373.

Kochan, Donald (2011): "Learning Research and Legal Education: A Brief Overview and Selected Bibliographical Survey”, Southwestern Law Review, vol. 40: pp. 449-471.

Lazo, Patricio (2011), "Formación Jurídica, Competencias y Métodos de Enseñanza: Premisas", Revista Ius et Praxis, año 17, № 1: pp. 249-262. 
MAnning, Bayless (1972): "La Enseñanza Jurídica Norteamericana. Evolución y Cambio. Tres Modelos", Boletín del Instituto de Docencia e Investigación Jurídicas, No 3: pp. 131-158.

Noble-Allgire, Alice M. (2002): "Desegregating the Law School Curriculum: How to Integrate More of the Skills and Values Identified by the MacCrate Reprot into a Doctrinal Course", Nevada Law Journal, vol. 3.32: pp. 32-60.

Orfield Gary y Kurlaender, Michael (2001): Diversity Challenged: Evidence on the Impact of Affirmative Action (Cambridge, Harvard Education Publishing Group, primera edición).

Peseta, Silvina (2008), "La Enseñanza del Derecho desde una Perspectiva Sociológico Jurídica”, Revista de Derecho Universidad Católica del Norte, No 2: pp. 133-156.

Salaburo, Pello (2003): Sistemas Universitarios en Europa y EE.UU. (Academia Europea de Ciencias y Artes, España, primera edición).

Salaburo, Pello (2007): La Universidad en la Encrucijada. Europa y EE.UU. (Academia Europea de Ciencias de España, Madrid, primera edición).

ShaKhtur, Alí (2007/2008), “El Rol del Abogado: ¿Negociador?”, Temas de Derecho, No 1 y 2: pp. 101-104.

Simpson, Ormond (2005): "The costs and benefits of student retention for students, institutions and Governments", Studies in Learning, Evaluation Innovation and Development, 2(3): pp. 34-43.

Solari, Enzo (2012), "El Currículo Chileno de Estudios Jurídicos", Revista de Derecho, Pontificia Universidad Católica de Valparaíso, vol. XXXIX, No 2: pp. 703-734.

Solari, Enzo (2015): "La filosofía jurídica y su enseñanza", Revista Chilena de Derecho, abril 2015, vol. 42, No.1: pp. 369-390.

SQuella, Agustín (1995): “Qué he aprendido enseñando filosofía del derecho?”, Gaceta Jurídica, No 178: pp. 7-34.

TAELI, Raquel et al. (2014): "Los aportes de paradigmas complejos y constructivistas para la enseñanza de la ciencia jurídica", Ius et Praxis, No 1: pp. 199-220.

Trow, Martin (1973): Problem in the Transition from Elite to Mass Higher Education (Berkeley, Carnegie Comision on Higher Education, primera edición).

Valenzuela, Rodrigo (2002): "El derecho y las humanidades", Revista de Derecho Universidad Austral de Chile, vol. XIII: pp. 41-44.

\section{JURISPRUDENCIA CITADA}

Regents of the UnIVERSity of California V. BakKe (1978): U.S. Supreme Court, July 28 (438 U.S. 256). Disponible en: https://supreme.justia.com/cases/federal/us/438/265/ case.html.

HOPWOOD V. TEXAS (1996): United States Court of Appeals, Firth Circuit, March 18 (78 F3d 932 5th Cir). Disponible en: https://scholarlycommons.law.wlu.edu/crsj/vol3/ iss $1 / 17$.

Grutter V. Bollinger (2003): U.S. Supreme Court, June 23 (539 U.S. 306). Disponible en: https://supreme.justia.com/cases/federal/us/539/306/case.html. 


\section{OTROS DOCUMENTOS}

Educational at Glance 2014 OCDE Indicators. Disponible en: http://www.keepeek. com/Digital-Asset-Management/oecd/education/education-at-a-glance-2014_eag2014en\#page7. Fecha de consulta: 14 de octubre de 2015. 